\title{
Tomosynthesis Is Equivalent to Computed Tomography for Evaluating Osseous Integration After Anterior Cruciate Ligament Reconstruction
}

\author{
Seikai Toyooka, M.D., Hironari Masuda, M.D., Nobuhiro Nishihara, M.D., \\ Naoya Shimazaki, M.D., Shuji Ando, Ph.D., Hirotaka Kawano, M.D., and \\ Takumi Nakagawa, M.D.
}

\begin{abstract}
Purpose: To compare tomosynthesis and computed tomography $(\mathrm{CT})$ for evaluating bone plug integration after anterior cruciate ligament (ACL) reconstruction with a bone-patellar tendon-bone (BPTB) graft. Methods: Data of consecutive adult patients who underwent ACL reconstruction with BPTB were analyzed. Bone integration between the bone plug and bone tunnel was evaluated by tomosynthesis and CT, which were both performed 3 months postoperatively. The obtained data for both modalities were reconstructed with slice thickness of $2 \mathrm{~mm}$. Evaluation of bone integration were separately performed using coronal- and sagittal-reconstructed images for the femur and tibia. The ratio of bone integration between the reconstructed slices in which bone grafting was involved, for both tomosynthesis and CT, was investigated by 2 blinded examiners. The equivalence of tomosynthesis to CT was tested by comparing the bone integration ratio for both modalities. The accuracy of diagnosing bone union using tomosynthesis and CT was also investigated. Results: The diagnostic accuracy of tomosynthesis and CT exceeded 80\%. Interobserver agreement of bone integration in the sagittal plane on the femoral side was 0.92 (intraclass correlation coefficient) for CT and 0.76 (intraclass correlation coefficient) for tomosynthesis. Conclusions: Although it showed poor reliability, tomosynthesis was equivalent to CT in evaluating bone plug integration after ACL reconstruction with BPTB. Level of Evidence: Level II, diagnostic study.
\end{abstract}

D igital tomosynthesis is an emerging imaging technique derived from radiographic tomography that has various clinical applications. Tomosynthesis images are reconstructed from a series of images with low-dose radiation exposure as the x-ray source moves in an arc or linear trajectory above the subject. It is currently being used in routine clinical practice for

From the Department of Orthopaedic Surgery, Teikyo University School of Medicine (S.T., H.M., N.N., N.S., H.K., T.N.); and Department of Information and Computer Technology Faculty of Engineering, Tokyo University of Science (S.A.), Tokyo, Japan.

The authors report that they have no conflicts of interest in the authorship and publication of this article. Full ICMJE author disclosure forms are available for this article online, as supplementary material.

Received June 26, 2019; accepted November 25, 2019.

Address correspondence to Takumi Nakagawa, M.D., Department of Orthopaedic Surgery, Teikyo University School of Medicine, 2-11-1, Kaga, Itabashi,Tokyo, 173-8605, Japan.E-mail:takumin-tky@umin.ac.jp

(C) 2020 Published by Elsevier on behalf of the Arthroscopy Association of North America. This is an open access article under the CC BY-NC-ND license

(http://creativecommons.org/licenses/by-nc-nd/4.0/).

2666-061X/19782

https://doi.org/10.1016/j.asmr.2019.11.006 imaging of the chest and breast, and it is beginning to be used for bone healing. ${ }^{1-5}$ Ha et al. $^{2}$ reported that tomosynthesis provided superior diagnostic information compared with other imaging methods during the postoperative evaluation of wrist fractures. Simoni et al. ${ }^{6}$ also reported that tomosynthesis and computed tomography (CT) detected a similar number of erosions in patients with rheumatic arthritis. Tomosynthesis is starting to attract attention from those who perform musculoskeletal diagnosis. ${ }^{1,7-10}$

Anterior cruciate ligament (ACL) reconstruction frequently is used to treat athletes and young patients who experience ligament injury. Although various grafts are considered for ACL reconstruction, the hamstring tendon or bone-patellar tendon-bone (BPTB) graft is mainly used for the procedure. In particular, after reconstruction with the BPTB graft, evaluation of bone integration between the bone plug and bone tunnel is important because it allows for the assessment of the graft condition and the planning of postoperative therapy. ${ }^{11}$ Standard radiography is the most commonly used examination for evaluating bone integration after ACL reconstruction with the BPTB graft ${ }^{12}$; however, it lacks 
sensitivity and specificity. Multidetector CT is an efficient tool that is also used for the evaluation of bone integration between the bone plug and bone tunnel. However, because of its cost and greater amount of radiation exposure, it is not used as frequently. Because tomosynthesis has lower radiation exposure and cost, it may replace CT as a highly reliable imaging method for general orthopaedic surgeons who need to evaluate integration. We aimed to determine whether tomosynthesis and $\mathrm{CT}$ have equivalent diagnostic value.

To the best of our knowledge, little is known about the diagnostic value of this new technology for bone plug integration after ACL reconstruction with the BPTB graft compared with $\mathrm{CT}$, which is the gold standard. The purpose of this study was to compare tomosynthesis and CT for evaluating bone plug integration after ACL reconstruction with a BPTB graft. It was hypothesized that an evaluation using tomosynthesis would be equivalent to an evaluation using CT after ACL reconstruction with the BPTB graft.

\section{Methods}

\section{Patients and Design}

The data of consecutive adult patients with isolated ACL injuries and ACL injuries with concomitant meniscal tears who underwent ACL reconstruction with the BPTB graft were analyzed prospectively. Patients underwent the procedure at a single institute between January 2017 and August 2017. The study protocol was approved by the institutional review board of the authors' institution, and all patients provided informed consent. The exclusion criteria were as follows: revision surgery, reconstruction with a hamstring tendon graft, and the inability to understand or comply with the study protocol. Each patient underwent both $\mathrm{CT}$ and tomosynthesis 3 months after surgery on the same day. Reconstructed data of both modalities were evaluated by 2 expert surgeons. A radiographic analysis was performed to investigate the whether the diagnostic value of tomosynthesis was equivalent to that of CT.

\section{Surgical Techniques}

All patients underwent ACL reconstruction with the anatomically rectangular tunnel BPTB graft, which used tourniquet control under general anesthesia according to the procedure reported by Shino et al. ${ }^{13}$ (Fig 1). Arthroscopy was performed to identify ACL tear and meniscal tears; if a meniscal tear was present, then a repair or meniscectomy was performed. The BPTB graft was harvested from the donor site and the shape of the bone graft on the patellar side was made rectangular. The size of the bone graft of the femoral side was 10 (side) $\times 15$ (vertical) $\times 5$ (thickness $) \mathrm{mm}$. The size of the bone graft of the tibial side was 10 (side) $\times 15$ (vertical) $\times 10$ (thickness) $\mathrm{mm}$. A bone tunnel was made from the far anteromedial portal. The apertures of both tunnels were made rectangular. For fixation of the graft, a $4 \times 12-\mathrm{mm}$ EndoButton (Smith $\delta$ Nephew, Andover, MA) was secured to the femoral bone plug using ULTRATAPE (Smith $\&$ Nephew) to place the distal end of the bone plug on the aperture of the femoral tunnel. Two leading sutures were used to pass the graft from the tibial tunnel to the femoral socket while maintaining the cancellous bone surface anteriorly. Femoral fixation was achieved with an EndoButton using the flip technique, and tibial fixation was performed using a Double Spike Plate system (MEIRA Corp., Nagoya, Japan) with the pull-out suture technique.

\section{Imaging Protocol}

Digital tomosynthesis was performed with a radiography fluoroscopy system (SONIALVISION Safire 17; Shimadzu Co., Kyoto, Japan) with a flat-panel detector system. The imaging conditions were as follows: $\mathrm{X}$-ray tube voltage, $47 \mathrm{kV}$; current, $160 \mathrm{~mA}$; data acquisition time for 74 projections, 2.5 or 5.0 seconds; frame rate,

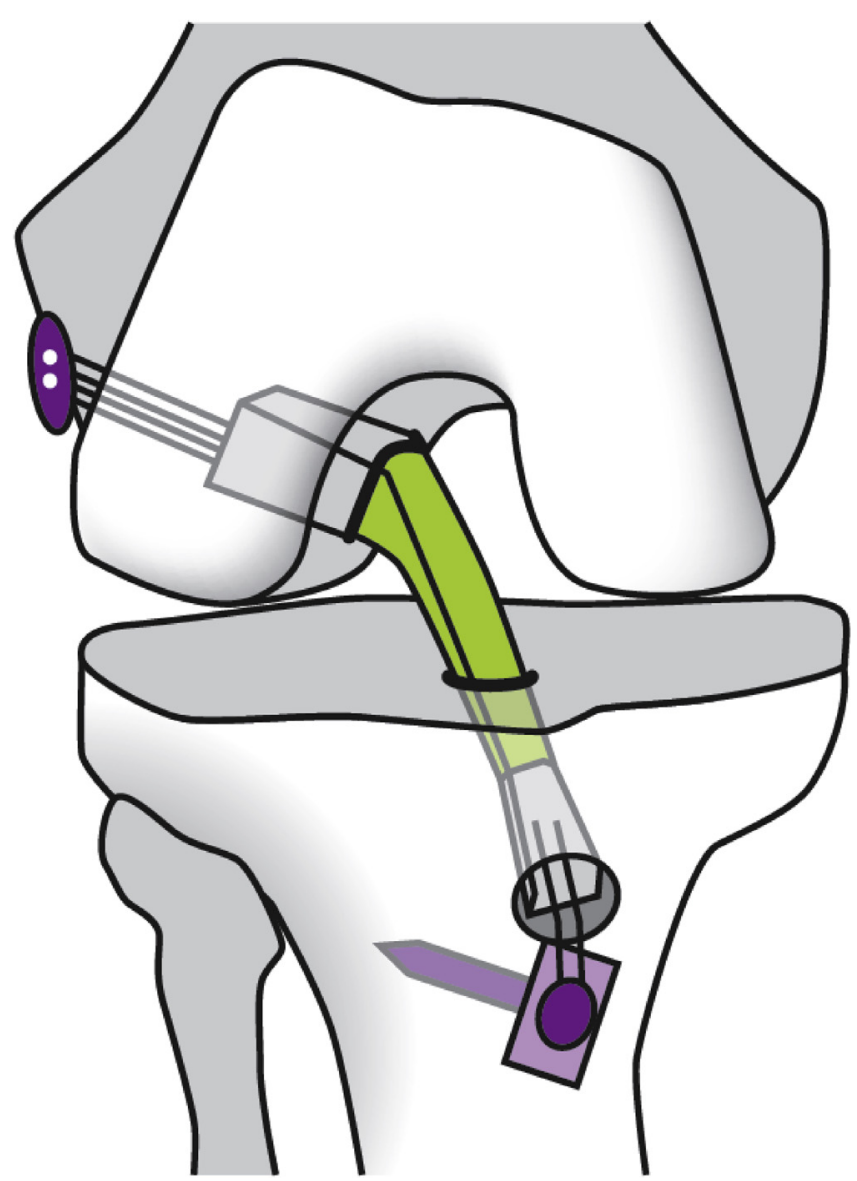

Fig 1. Schema of the anatomically rectangular anterior cruciate ligament reconstruction in a right knee. 
30 or 15 frames per second; number of reconstruction images, 21; and field of view, 12 inches.

CT (Toshiba Aquilion; Toshiba Medical Systems Corporation, Otawara, Japan) was performed using the standard bone CT protocol with $0.5-\mathrm{mm}$ axial sections in 3 planes, with a tube voltage of $120 \mathrm{kV}$. The data obtained using both modalities were reconstructed with slice thickness of $2 \mathrm{~mm}$ in the coronal and sagittal planes.

\section{Radiographic Analysis}

Images were evaluated independently by 2 expert orthopaedic surgeons with 14 years and 21 years of clinical experience, respectively; they were blinded to the clinical and patient data. The evaluation was conducted using a PACS workstation (V5 Impax, Agfa HealthCare, Mortsel, Belgium). The images were anonymized and displayed in random order.

The examiners determined how many slices of the graft had experienced integration among the slices in which bone grafting was involved. A slice was defined to have achieved bone integration if there was bone trabeculae continuity between the bone plug and bone tunnel $^{14}$ (Fig 2). The sagittal plane on the femoral side and the coronal plane on the tibial side were investigated in this study using methods described in a previous study ${ }^{11}$ (Fig 3). The ratio of bone integration was compared between both modalities by both surgeons.

Although the time required for tomosynthesis to evaluate bone graft integration was not established using a comparison of both modalities, we evaluated CT and tomosynthesis data at 3 months postoperatively during this study. Masuda et al. ${ }^{11}$ reported that bone grafts were $100 \%$ healed at 5 months postoperatively; however, only $50 \%$ were healed at 3 months. Therefore, a $50 \%$ integration rate seemed suitable for this study.
To confirm the validity of tomosynthesis compared with that of CT, the primary outcome of both modalities was evaluated by an equivalence test. We investigated the $95 \%$ confidence interval of the bone integration ratio difference between both modalities for each surgeon's measurements. The difference in the bone integration ratio was obtained by subtracting the integration ratio of tomosynthesis from that of CT. When comparing tomosynthesis with $\mathrm{CT}$, a diagnostic error within $15 \%$ was tentatively defined as the equivalence margin. If the $95 \%$ confidence interval of the difference between both modalities was within the equivalence margin, then the validity of tomosynthesis was considered equivalent to that of CT.

In addition, the diagnostic accuracy of tomosynthesis compared with that of CT was investigated as a secondary outcome. The graft was considered to achieve bone union when the integration rate exceeded $50 \%$. The sensitivity, specificity, positive predictive value (PPV), negative predictive value (NPV), and accuracy of tomosynthesis compared to that of CT were investigated. Moreover, interobserver agreement was assessed with intraclass correlation coefficient (ICC) values.

\section{Statistical Analysis}

The sample size estimation of paired data was performed before the study using the data of a pilot study. In that pilot study, the average difference between the 2 groups was $0.1(10 \%)$, therefore, it was impossible to set the equivalence margin within $10 \%$. As a result, $15 \%$ was the lowest value that could be set as the equivalence margin at this study. Although the range of acceptable diagnostic error regarding integration after ACL with the BPTB graft is unknown, a difference within $15 \%$ of the evaluation margin of bone integration between both groups was defined as acceptable based on clinical experience. A sample size of 27

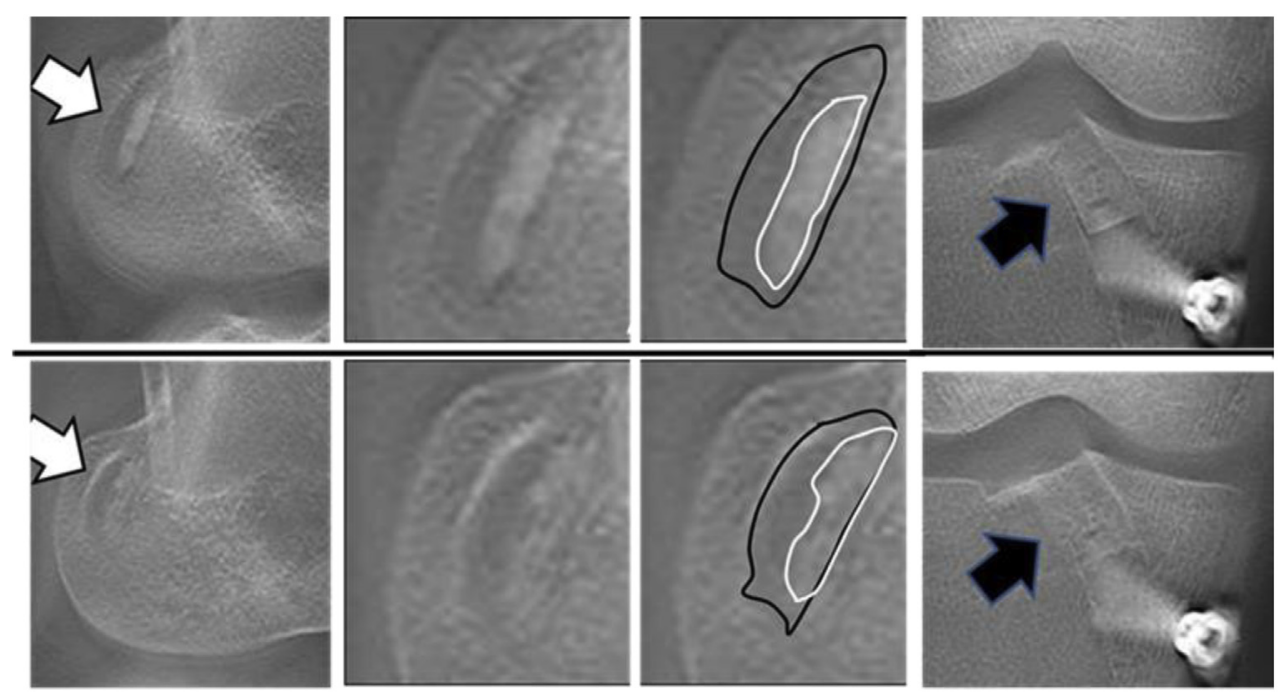

Bone integration (-)

Fig 2. Sagittal plane tomosynthesis on the femoral side in a right knee. Bone integration was negative in the upper panels; however, the bone plug was integrated in the lower panels. The white arrows indicate the femoral tunnel in the sagittal plane. The black arrows indicate the tibial tunnel in the coronal plane. 


\section{CT Tomosynthesis}


Fig 3. Sample images of computed tomography (CT) and tomosynthesis in the coronal and sagittal planes of the femur and tibia in a left knee. subjects was required in each group, to detect equivalence at an alpha level of 0.05 with a power of $80 \%$.

Equivalence tests of paired data of both modalities were performed using Minitab 18 statistical software (Minitab Inc., State College, PA). The paired $t$ test, which compared the integration rates of both modalities, was performed and the ICC was calculated using SPSS, version 12, software (SPSS Inc., Chicago, IL). A $P$ value $<.05$ was considered statistically significant. Rates of sensitivity and specificity for each modality were calculated based on the measurements performed by each surgeon; accuracy, PPV, NPV, and odds ratios also were determined.

\section{Results}

The imaging data of 27 patients were reviewed (CT, $\mathrm{n}=27$; tomosynthesis, $\mathrm{n}=27$ ). Preoperative patient characteristics are shown in Table 1.

The bone integration rates of the bone plug and bone tunnel as evaluated by the 2 surgeons are shown in Figure 4 . The integration rate of the femoral side in the sagittal plane 3 months after surgery was approximately 60\% (60\%: surgeon 1 using CT: $59 \%$ : surgeon 1 using tomosynthesis; $57 \%$ : surgeon 2 using CT; $60 \%$ : surgeon 2 using tomosynthesis). The integration rate of the tibial side in the coronal plane 3 months after surgery was approximately $88 \%$ (88\%: surgeon 1 using CT; $87 \%$ : surgeon 1 using tomosynthesis; $88 \%$ : surgeon 2 using CT; $88 \%$ : surgeon 2 using tomosynthesis). The integration rate on the femoral side in the sagittal plane as assessed by surgeon 1 was significantly different when determined using CT $(0.60 \pm 0.28)$ and tomosynthesis $(0.69 \pm 0.29, P=.025)$. However, no significant difference was found on the tibial side in the coronal plane by surgeon 1, and no significant difference was found on the femoral side in the sagittal plane or on the tibial side in the coronal plane by surgeon 2 .

Regarding the equivalence test, the $95 \%$ confidence interval of the difference between CT and tomosynthesis for all evaluations by both surgeons was within the equivalence margin $(-0.15$ to 0.15$)$; therefore, equivalence was proven (Fig 5). Evaluations by both surgeons of the bone integration rate yielded greater values when using tomosynthesis than when using CT in the sagittal plane on the femoral side. However, the bone integration rate observed with $\mathrm{CT}$ was greater

Table 1. Preoperative Patient Characteristics

\begin{tabular}{ll}
\hline \multicolumn{1}{c}{ Parameter } & \\
\hline Male/female & $16 / 11$ \\
Right/left & $11 / 16$ \\
Age, y & $27.9(16-43)$ \\
Height, cm & $171.2 \pm 10.1$ \\
Weight, kg & $71.2 \pm 5.2$ \\
Body mass index & $24.5 \pm 4.6$ \\
Athlete/nonathlete & $18 / 9$ \\
KT-1000, mm & $4.6 \pm 2.5$ \\
\hline
\end{tabular}

Values are presented as numbers, mean \pm standard deviation, or mean (range). 
A

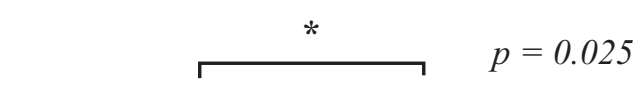

1

0.8

0.6

0.4

0.2

0

Fig 4. The union rates according to surgeon 1 and surgeon 2. (A) Surgeon 1. (B) Surgeon 2. *Significant difference. (CT, computed tomography; n.s., not significant.)

Femur sagittal

$p=0.025$

1

(1)
B

Femur sagittal

Tibia coronal

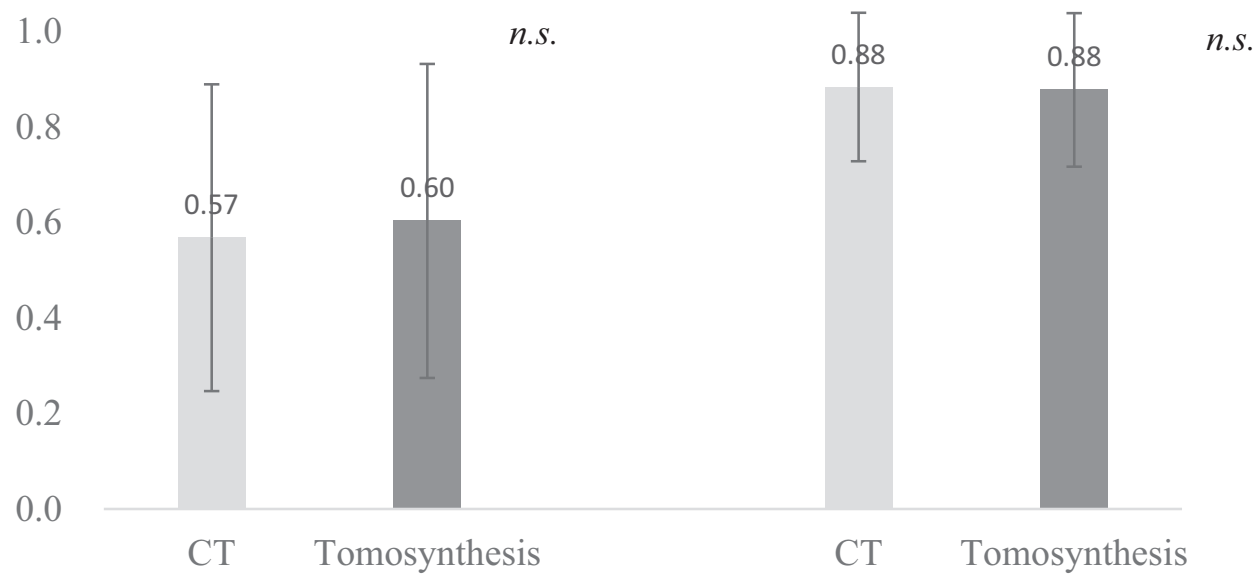

Tibia coronal

n.s.

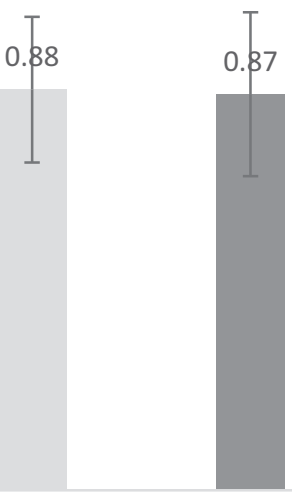

CT Tomosynthesis than that observed with tomosynthesis in the coronal plane on the tibial side.

Sensitivity, specificity, PPV, NPV, and accuracy are shown in Table 2. The accuracy of tomosynthesis compared with that of CT for all measurements by both surgeons exceeded $80 \%$ when the graft was defined as achieving bone union with integration rates that exceeded $50 \%$.

Interobserver agreement of bone integration in the sagittal plane on the femoral side was 0.92 (ICC) for CT and 0.76 (ICC) for tomosynthesis. Interobserver agreement in the coronal plane of the tibial side was 0.74 (ICC) for CT and 0.73 (ICC) for tomosynthesis. 
A

$(\mathrm{LEL}=$ Lower Equivalence Limit, UEL = Upper Equivalence Limit $)$

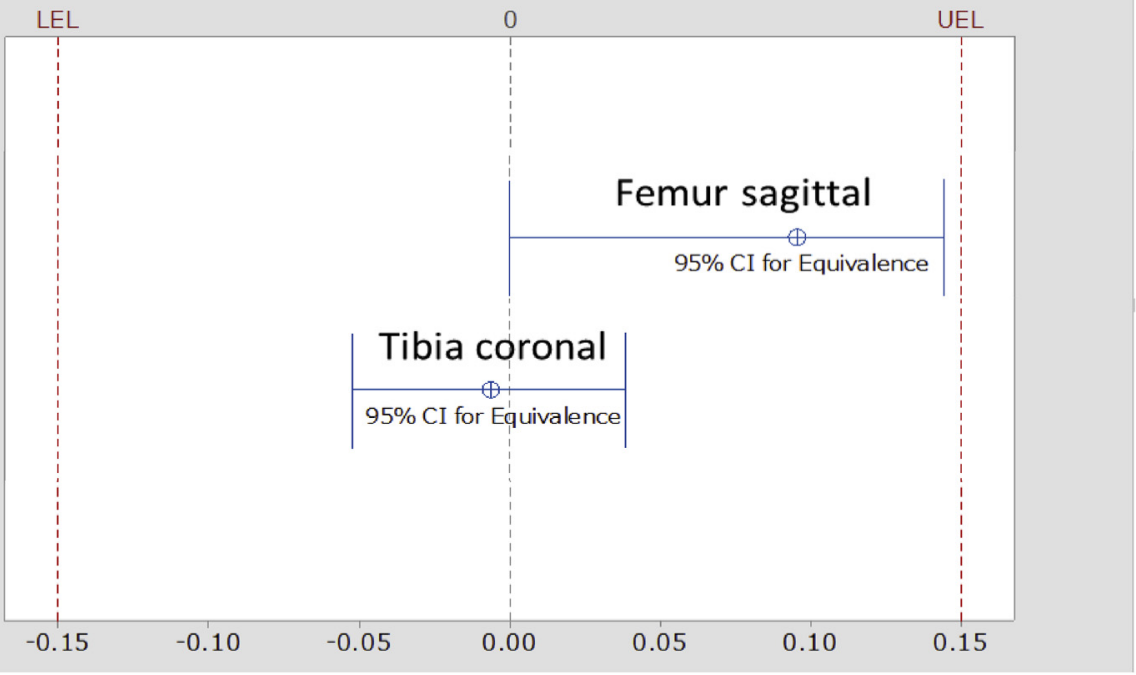

B

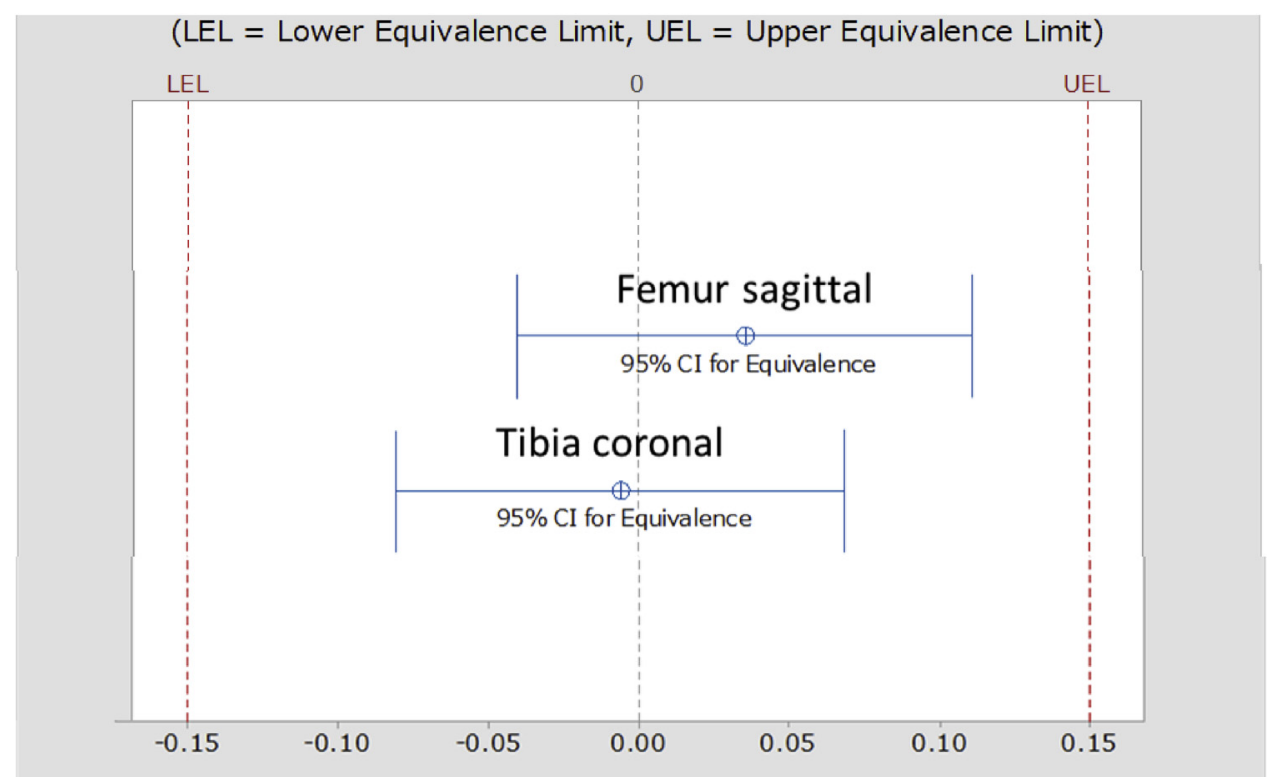

Fig 5. Equivalence test: union rates of tomosynthesis and CT. (A) Surgeon 1. (B) Surgeon $2.95 \%$ CI for equivalence of the mean data of tomosynthesis and the mean data of CT. The CI was within the equivalence interval of -0.15 and 0.15 , suggesting equivalence. (CI, confidence interval; CT, computed tomography; LEL, lower equivalence limit; UEL, upper equivalence limit.) sagittal plane as assessed by surgeons 1 and 2, the inverse was true for the tibial side in the coronal plane. This might have occurred because the resolution of tomosynthesis was lower than that of CT. Therefore, tomosynthesis might have a higher or lower integration rate than $\mathrm{CT}$, depending on the imaging site and conditions.

Most previous diagnostic comparison studies of tomosynthesis and CT such as those involving lung nodule findings or breast cancer diagnoses, investigated

Table 2. Accuracy of Tomosynthesis Versus Computed Tomography

\begin{tabular}{clccccc}
\hline Reader & \multicolumn{1}{c}{ Site } & Sensitivity $(\%)$ & Specificity $(\%)$ & PPV $(\%)$ & NPV $(\%)$ & Accuracy $(\%)$ \\
\hline Surgeon 1 & Femur, sagittal & 94 & 64 & 79 & 88 \\
& Tibia, coronal & 96 & 100 & 100 & 67 \\
Surgeon 2 & Femur, sagittal & 79 & 100 & 100 & 67 \\
& Tibia, coronal & 96 & 100 & 100 & 67 \\
\hline
\end{tabular}

NPV, negative predictive value; PPV, positive predictive value. 
only the sensitivity or specificity of tomosynthesis and used CT as the gold standard. ${ }^{15-17}$ The equivalence test was used in this study to confirm the validity of tomosynthesis. The equivalence test has been strongly recommended for demonstrating the comparability of treatment effects in various research fields, including medical studies. ${ }^{18-20}$ This test is a variation of hypothesis tests used to draw statistical inferences from observed data and originated from the field of pharmacokinetics. One application of the test is to show that a new drug that is less expensive than the existing available alternatives works just as well. Equivalence tests are used to calculate a confidence interval based on an observed effect size and reject effects that are more extreme than the equivalence margin when the confidence interval does not overlap with the equivalence margin. In 2-sided tests, upper and lower equivalence margins are specified. In noninferiority trials, which aim to test the hypothesis that a new technique is not worse than an existing one, only a lower equivalence margin is prespecified; therefore, a noninferiority trial was not suitable for this study.

In equivalence tests, the null hypothesis can be defined as an effect large enough to be deemed effective, as specified by an equivalence margin. In this study, by defining a $15 \%$ diagnostic error as the equivalence margin, the diagnostic error of tomosynthesis compared with that of CT could be quantitatively evaluated. In addition, in our study, the diagnostic accuracy of tomosynthesis was investigated using the methods of previous studies of lung nodule findings or breast cancer diagnosis. ${ }^{16,21,22}$

The image condition and the timing of capture were not the same for both modalities according to a previous study that compared the usefulness of tomosynthesis and CT. ${ }^{23}$ However, the present study compared $\mathrm{CT}$ and tomosynthesis performed on the same day. The data obtained using both modalities were reconstructed with slice thickness of $2 \mathrm{~mm}$ in the coronal and sagittal planes. Therefore, the observer could judge both sets of reconstruction data equally.

Using tomosynthesis instead of CT has many advantages. First, the examination time is shorter; the data acquisition time for projections was only 2.5 to 5.0 seconds. Second, the cost is lower. Although the cost of tomosynthesis is twice that of radiography, it is one-third that of CT and one-fourth that of magnetic resonance imaging in Japan. Quaia et al. ${ }^{24}$ stated that the average per-patient costs of tomosynthesis and CT were $€ 41.55$ and $€ 113.66$, respectively, in Italy. Third, the influence of metal equipment-related artifacts is reduced. ${ }^{25}$ Fourth, the associated radiation dose is lower than that of CT; however, the radiation exposure of tomosynthesis is twice that of radiography. Koyama et al. ${ }^{26}$ stated that the effective dose during a typical tomosynthesis examination of the hip joint was $0.82 \mathrm{mSv}$; however, the effective dose during routine $\mathrm{CT}$ examination in the same location was $10.5 \mathrm{mSv}$, which is more than 10 times that of tomosynthesis.

Although this study only investigated graft integration, there are several possible uses for tomosynthesis, which is an emerging imaging technique. It may be useful for evaluating transposition of the bone graft, bone absorption in the bone tunnel, and tunnel enlargement after ACL reconstruction not only with the BPTB graft but also with the hamstring graft. Furthermore, this study demonstrated the usefulness of tomosynthesis even for bone grafts as small as $15 \mathrm{~mm}$. Tomosynthesis also may be useful for small bone grafts in other parts of the body. Further research is needed to confirm the usefulness of tomosynthesis.

\section{Limitations}

This study had some limitations. First, the equivalence margin was not established for the comparison between CT and tomosynthesis. Fifteen percent was selected because it was not different from that used in actual clinical practice, which uses a diagnosis error of $15 \%$ (equivalence margin of 0.15 ), and because it was feasible for the number of cases in this study. However, as a result of setting equivalence margin as $15 \%$, we could show that the equivalence of tomosynthesis to CT was exactly $10 \%$ to $15 \%$. Second, the use of digital tomosynthesis to evaluate bone integration is relatively poorly reported, and no definite criteria have been established.

\section{Conclusions}

Although it showed poor reliability, tomosynthesis was equivalent to $\mathrm{CT}$ in evaluating bone plug integration after ACL reconstruction with BPTB.

\section{Acknowledgments}

The authors thank Dr. Asuka Nemoto for advice regarding statistical experiments.

\section{References}

1. Blum A, Noel A, Regent D, Villani N, Gillet R, Gondim Teixeira P. Tomosynthesis in musculoskeletal pathology. Diagn Interv Imaging 2018;99:423-441.

2. Ha AS, Lee AY, Hippe DS, Chou SH, Chew FS. Digital tomosynthesis to evaluate fracture healing: Prospective comparison with radiography and CT. AJR Am J Roentgenol 2015;205:136-141.

3. Machida H, Yuhara T, Sabol JM, Tamura M, Shimada Y, Ueno E. Postoperative follow-up of olecranon fracture by digital tomosynthesis radiography. Jpn J Radiol 2011;29: 583-586.

4. Petraszko A, Siegal D, Flynn M, Rao SD, Peterson E, van Holsbeeck M. The advantages of tomosynthesis for evaluating bisphosphonate-related atypical femur fractures 
compared to radiography. Skeletal Radiol 2016;45: 615-623.

5. Tateiwa D, Outani H, Iwasa S, et al. Atypical femoral fracture associated with bone-modifying agent for bone metastasis of breast cancer: A report of two cases. J Orthop Surg (Hong Kong) 2017;25:2309499017727916.

6. Simoni P, Gerard L, Kaiser MJ, et al. Use of tomosynthesis for detection of bone erosions of the foot in patients with established rheumatoid arthritis: Comparison with radiography and CT. AJR Am J Roentgenol 2015;205:364-370.

7. Canella C, Philippe P, Pansini V, Salleron J, Flipo RM, Cotten A. Use of tomosynthesis for erosion evaluation in rheumatoid arthritic hands and wrists. Radiology 2011;258:199-205.

8. Hayashi D, Xu L, Gusenburg J, et al. Reliability of semiquantitative assessment of osteophytes and subchondral cysts on tomosynthesis images by radiologists with different levels of expertise. Diagn Interv Radiol 2014;20: 353-359.

9. Levakhina YM, Duschka RL, Vogt FM, Barkhausen J, Buzug TM. A dual-axis tilt acquisition geometry for digital musculoskeletal tomosynthesis. Phys Med Biol 2013;58: 4827-4848.

10. Song Y, Lee S, Lee BG, Joo YB, Song SY. The diagnostic reproducibility of tomosynthesis for the correlation between acromiohumeral distance and rotator cuff size or type. Korean J Radiol 2018;19:417-424.

11. Masuda H, Taketomi S, Inui $\mathrm{H}$, et al. Bone-to-bone integrations were complete within 5 months after anatomical rectangular tunnel anterior cruciate ligament reconstruction using a bone-patellar tendon-bone graft. Knee Surg Sports Traumatol Arthrosc 2018;26:3660-3666.

12. Kim M, Choi YS, Kim H, Choi NH. Postoperative evaluation after anterior cruciate ligament reconstruction: Measurements and abnormalities on radiographic and CT imaging. Korean J Radiol 2016;17:919-930.

13. Shino K, Nakata K, Nakamura N, et al. Rectangular tunnel double-bundle anterior cruciate ligament reconstruction with bone-patellar tendon-bone graft to mimic natural fiber arrangement. Arthroscopy 2008;24:1178-1183.

14. Corrales LA, Morshed S, Bhandari M, Miclau T 3rd. Variability in the assessment of fracture-healing in orthopaedic trauma studies. J Bone Joint Surg Am 2008;90: 1862-1868.

15. Gilbert FJ, Tucker L, Gillan MG, et al. Accuracy of digital breast tomosynthesis for depicting breast cancer subgroups in a UK retrospective reading study (TOMMY Trial). Radiology 2015;277:697-706.

16. Grosso M, Priotto R, Ghirardo D, et al. Comparison of digital tomosynthesis and computed tomography for lung nodule detection in SOS screening program. Radiol Med 2017; 122:568-574.

17. Shim SS, Oh YW, Kong KA, Ryu YJ, Kim Y, Jang DH. Pulmonary nodule size evaluation with chest tomosynthesis and CT: A phantom study. Br J Radiol 2015;88: 20140040.

18. Lakens D. Equivalence tests: A practical primer for t tests, correlations, and meta-analyses. Soc Psychol Personality Sci 2017;8:355-362.

19. Rosas-Peralta M, Santos-Martinez LE, MaganaSerrano JA, Valencia-Sanchez JS, Garrido-Garduno M, Perez-Rodriguez G. Methodology for superiority versus equivalence and non-inferior clinical studies. A practical review [in Spanish]. Rev Med Inst Mexicano del Seguro Soc 2016;54:344-353.

20. Shieh G. Exact power and sample size calculations for the two one-sided tests of equivalence. PLoS One 2016;11: e0162093.

21. Gong X, Glick SJ, Liu B, Vedula AA, Thacker S. A computer simulation study comparing lesion detection accuracy with digital mammography, breast tomosynthesis, and cone-beam CT breast imaging. Med Phys 2006;33:1041-1052.

22. Nelson G, Wu M, Hinkel C, et al. Improved targeting accuracy of lung tumor biopsies with scanning-beam digital $x$-ray tomosynthesis image guidance. Med Phys 2016;43:6282.

23. Ottenin MA, Jacquot A, Grospretre O, et al. Evaluation of the diagnostic performance of tomosynthesis in fractures of the wrist. AJR Am J Roentgenol 2012;198:180-186.

24. Quaia E, Grisi G, Baratella E, et al. Diagnostic imaging costs before and after digital tomosynthesis implementation in patient management after detection of suspected thoracic lesions on chest radiography. Insights Imaging 2014;5:147-155.

25. Gillet R, Teixeira P, Bonarelli C, et al. Comparison of radiographs, tomosynthesis and CT with metal artifact reduction for the detection of hip prosthetic loosening. Eur Radiol 2019;29:1258-1266.

26. Koyama S, Aoyama T, Oda N, Yamauchi-Kawaura C. Radiation dose evaluation in tomosynthesis and C-arm cone-beam CT examinations with an anthropomorphic phantom. Med Phys 2010;37:4298-4306. 